\title{
Decline and revival of McCollough effects following inspection of achromatic gratings
}

\author{
D. SKOWBO and N. CLYNES \\ Colby College, Waterville, Maine 04901
}

\begin{abstract}
We have investigated the reduction in estimated strength of McCollough effects that results from inspection of achromatic gratings. Longer inspection times were found to produce greater decreases in our assessments than shorter times. Following a rest period, increments in our strength index were observed, with greatest gains associated with shortest inspection times, and least gains with longest times. Our results can be construed as supportive of a learning model of McCollough effects, or, alternatively, as suggesting the existence of achromatic McCollough effects.
\end{abstract}

Aftereffects of color which are contingent on the orientation of lines were first described by McCollough in 1965 and now are often known by her name. They are commonly produced by having subjects view chromatic grating patterns; subsequently, black and white "test" patterns appear tinted with colors that are roughly complementary to those which had been presented with each grating orientation.

An outstanding feature of McCollough effects (MEs) is their long duration, and several recent studies have examined the decay characteristics of MEs (e.g., MacKay \& MacKay, 1974; Riggs, White, \& Eimas, 1974). Attention has also been given to procedures which influence decay rate (MacKay \& Mackay, 1975), especially those that appear to promote an unusually rapid decline (Skowbo, Gentry, Timney, \& Morant, 1974). The finding that estimates of $\mathrm{ME}$ strength are reduced by inspection of achromatic gratings was first reported by Skowbo et al (1974) and has since been investigated by White and Graves (Note 1). Results of the latter study indicated that, in time, there is a partial recovery of strength. In addition, White and Graves showed that inspection of achromatic gratings prior to ME induction reduced subsequent ME assessments.

The importance to theory of these findings led us to a parametric investigation of the recovery of MEs whose estimated strength has been reduced by exposure to achromatic patterns. The independent variables in our study were time of exposure to black and white gratings, and time subsequently allowed as a rest period before testing for recovery.

\section{METHODS}

Two trained observers with normal color vision were used. In all conditions, the procedure had three basic steps. First, MEs

This research was supported by a faculty research grant from Colby College. were induced by having observers monocularly (with the left eye) view alternating horizontal and vertical green (Wratten 53) and magenta (Wratten 32) gratings ( 5 cycles/deg). The gratings alternated every $10 \mathrm{sec}$ for a total of $5 \mathrm{~min}$. Next, the observers viewed alternating horizontal and vertical achromatic gratings. Again, the patterns changed every $10 \mathrm{sec}$; exposure durations were 10,20 , or $30 \mathrm{~min}$. Finally, the observers had a rest period, during which the left eye was covered with a black patch; these periods lasted 10,20 , or $30 \mathrm{~min}$. There were, then, nine combinations of achromatic grating exposure times and rest period times. Each experimental session consisted of one combination, and each observer was tested on each combination twice.

Appearance of color on a test pattern was evaluated before and after ME induction, after exposure to the achromatic gratings, and after the rest period. A projection colorimeter provided a homogeneous chromatic field which was viewed with the right eye; it was adjusted by the observer to match effects seen on the test pattern with the left eye. Each testing block consisted of eight matches, in an ABBA sequence, to vertical and horizontal portions of the test grating. Prior to each block was a 5-min period of binocular light adaptation.

Both observers were able to match their effects with mixtures of lights transmitted by two Wratten filters (CC30M and CC50G). All matches could thus be located in CIE space along a straight line connecting two points with the coordinates $x=.435, y=.368$ and $x=.416, y=.466$.

Since two aftereffect colors were assessed in each group of matches, an indication of ME strength could be derived from considering the measurements either independently or jointly. We derived a simple index by measuring the distance in CIE space between matches to vertical and horizontal portions of the test pattern. For any given session, this distance was near zero between preadaptation matches, maximal between matches made immediately after ME induction, and intermediate between the two remaining pairs of assessments.

\section{RESULTS}

There were two main findings. One confirms and extends earlier reports (Skowbo et al., 1974; White \& Graves, Note 1) that exposure to achromatic gratings reduces assessed strength of MEs. Distance between matches was reduced from maximum by amounts that varied slightly with exposure times: collapsing across observers and rest-period-time conditions, to about $47 \%$ of maximum for $10 \mathrm{~min}$, about $42 \%$ for $20 \mathrm{~min}$, and about $40 \%$ for $30 \mathrm{~min}$. 


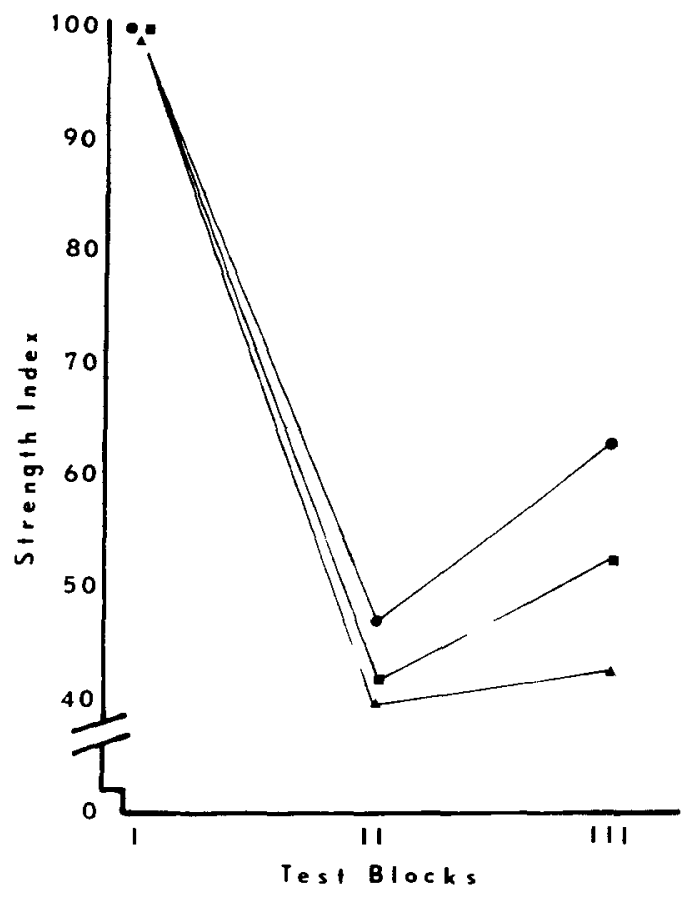

Figure 1. ME strength assessed in three testing blocks. The ordinate represents normalized distance in CIE space between matches to vertical and horizontal portions of a test pattern. The abcissa represents testing blocks carried out after ME induction (I), after inspection of achromatic patterns (II), and after a rest period (III). Achromatic grating exposure times are indicated by the symbols - for $10 \mathrm{~min}$, for $20 \mathrm{~min}$, and $\Delta$ for $30 \mathrm{~min}$.

The second major finding concerned increases in estimated aftereffect strength following rest periods. For both observers, greatest gains in the index were found when achromatic grating exposure time had been $10 \mathrm{~min}$, and least gains followed 30 -min inspections. No systematic differences in amounts of recovery were found to be associated with rest-period times.

Figure 1 depicts these findings. The ordinate is a normalization of our index of ME strength. For a given session, any initial color bias in preadaptation matches was subtracted from the distance between matches made after $\mathrm{ME}$ induction. The resulting distance was taken as maximum, or $100 \%$, and subsequent distances between matches were expressed as percentages of it. The abscissa indicates testing blocks: Block I is initial, or maximum, MEs; Block II is effects measured after achromatic pattern inspection; and Block III is effects assessed after rest periods. (Though testing blocks followed each other in temporal order, it should be kept in mind that the abscissa does not indicate linear time.) Different symbols indicate different times of exposure to the gratings, each point representing an average across observers and rest period times.

\section{DISCUSSION}

There are several ways to interpret the finding that ME strength appears to decline following achromatic grating inspection. One is to suppose that viewing an achromatic pattern disturbs the appearance of color associated with line orientation in the same way that extinction trials disturb the appearance of learned responses. Such an account is, of course, based on a classical conditioning model of MEs. It does not readily lend itself to the White and Graves (Note 1) finding that such inspections can diminish MEs in advance of their establishment. ${ }^{1}$

Another possible interpretation is that MEs appear to weaken because an "achromatic McCollough effect"' builds up, reducing the assessed strength of the chromatic effect (White, Note 2). This account readily encompasses the White and Graves finding. Acromatic MEs per se have yet to be demonstrated, but if they did exist, we might expect their acquisition and decay characteristics to qualitatively resemble those of the chromatic effects. For example, Riggs et al. (1974) found aftereffect strength to be roughly linearly related to $\log$ inspection time-there are, in other words, diminishing returns to increasing exposure duration. Our finding that different times of inspecting achromatic gratings produced only small differences in estimates of strength reduction would be consistent with the possibility that our exposure times fell on a portion of a hypothetical acquisition function that increased but little. Applying this interpretation to our other main finding, the chromatic effect may appear to revive when the achromatic one has decayed noticeably. Predicting net assessments of combined chromatic effects, as their decay proceeds, has been illustrated by White (1976). However, since there are, of course, no quantitative descriptions of achromatic effects, differences in amounts of recovery associated with our inspection and rest period times would be difficult to anticipate.

A learning account would predict that less recovery be observed as the number of "extinction" trials increased; this was observed. However, such a model might also suggest that longer rest periods be associated in some way with larger amounts of recovery, and we did not find such a relationship.

Differences between these two theoretical accounts tend to break down when extinction is considered as a matter of learning not to respond-the response, in this case, being whatever processes produce the appearance of color. Nevertheless, the questions of why exposure to achromatic gratings lowers ME strength estimates and why the assessments recover will remain unresolved until more is known about the possible existence of achromatic McCollough effects. 


\section{REFERENCE NOTES}

1. White, K. D., \& Graves, A. L. Influence of achromatic inspection on McCollough effects. Paper presented at the meeting of the Association for-Research in Vision and Ophthalmology, Sarasota, Florida, May 1976.

2. Personal communication with K. D. White, 1976.

\section{REFERENCES}

Hall, J. J. Classical conditioning and instrumental learning. New York: Lippincott, 1976.

Mackay, D. M., \& MacKay, V. The time course of the McCollough effect and its physiological implications. Journal of Physiology, 1974, 237, 38-39P.

MACKAY, D. M., \& MACKAY, V. What causes decay of patterncontingent chromatic aftereffects? Vision Research, 1975, 15, $462-464$.

Riggs, L. A., White, K. D., \& Eimas, P. D. Establishment and decay of orientation-contingent aftereffects of color. Perception \& Psychophysics, 1974, 16, 535-542.

Skowbo, D., Gentry, T., Timney, B., \& Morant, R. B. The McCollough effect: Influence of several kinds of visual stimulation on decay rate. Perception \& Psychophysics, 1974, 16, 47-49.

WHITE, K. D. Studies of orientation-contingent color aftereffects. Unpublished doctoral thesis, Brown University, 1976.

\section{NOTE}

1. The phenomenon of latent inhibition could, however, be invoked as an analogy. Hall (1976) contains a summary of relevant literature.

(Received for publication August 27, 1976; accepted October 22, 1976.) 